\title{
Terracini loci and homogeneous spaces
}

\section{Terracini Loci et espaces homogènes}

\author{
Edoardo Ballico*
}

Dept. of Mathematics, University of Trento, 38123 Povo (TN), Italy

ballico@science.unitn.it

ABSTRACT. We study the linear dependence of disjoint unions of double points of an integral and non-degenerate variety $X \subset \mathbb{P}^{r}$. Such sets are called Terracini loci. Our main results are for Segre-Veronese embeddings and a few other homogeneous spaces. To study the minimal number of such double points which are linearly dependent, it is useful to study the minimal degree curves contained in $X$. We give an example (the Segre embedding of $\mathbb{P}^{1} \times \mathbb{P}^{1}$ ) in which these curves are not sufficient to describe these Terracini loci.

2010 Mathematics Subject Classification. 14 N05.

KEYWORDS. Terracini's lemma, homogeneous spaces, secant varieties, zero-dimensional schemes, tangent spaces.

\section{Introduction}

Let $X \subset \mathbb{P}^{r}$ be an integral and non-degenerate variety defined over an algebraically closed field $\mathbb{K}$. Set $n:=\operatorname{dim} X$. Let $X_{\text {reg }}$ denote the set of all smooth points of $X$. For any $p \in X_{\text {reg }}$ let $(2 p, X)$ denote the closed subscheme of $X$ with $\left(\mathcal{I}_{p, X}\right)^{2}$ as its ideal sheaf. The scheme $(2 p, X)$ is zero-dimensional, $\operatorname{deg}(2 p, X)=\operatorname{dim} X+1$ and $(2 p, X)_{\text {red }}=\{p\}$. For any finite set $S \subset X_{\text {reg }}$ set $(2 S, X):=\cup_{p \in S}(2 p, X)$ and $\delta(2 S, X):=h^{1}\left(\mathbb{P}^{r}, \mathcal{I}_{(2 S, X)}(1)\right)$. For any positive integer $x$ let $S\left(X_{\text {reg }}, x\right)$ denote the set of all finite subsets of $X_{\text {reg }}$ with cardinality $x$. The Terracini locus $\mathbb{T}_{1}(X, x)$ is the set of all $S \in S\left(X_{\text {reg }}, x\right)$ such that $h^{0}\left(\mathcal{I}_{(2 S, X)}(1)\right)>0$ and $h^{1}\left(\mathcal{I}_{(2 S, X)}(1)\right)>0$.

Fix a finite set $S \subset X_{\text {reg. }}$. The set $S$ define the following function

$$
t_{X, S}: X_{\text {reg }} \backslash S \rightarrow \mathbb{N}
$$

by the formula $t_{X, S}(u):=\delta(2(S \cup\{u\}), X)$ for all $u \in X_{\text {reg }} \backslash S$. The sets $t_{X, S}^{-1}(x), x \in \mathbb{N}$, form the first Terracini stratification of the embedding of the pair $(X, S)$ in $\mathbb{P}^{r}$. By the semicontinuity theorem for cohomology each stratum of this stratification is locally closed in $X_{\text {reg. }}$.

For any $p \in X_{\text {reg }}$ let $T_{p} X \subset \mathbb{P}^{r}$ denote the Zariski tangent space of $X$ at $p$. A tangent vector or an arrow of $X_{\text {reg }}$ is a connected zero-dimensional scheme $v \subset X_{\text {reg }}$ such that $\operatorname{deg}(v)=2$. Let $v \subset X_{\text {reg }}$ be an arrow. Since $v$ is connected and zero-dimensional, $v_{\text {red }}$ is a point of $X_{\text {reg. Any arrow with } p \text { as its }}$ reduction is contained in $T_{p} X$ and $T_{p} X$ is spanned by all arrows with $p$ as its reduction. This is equivalent to say that $\langle(2 p, X)\rangle=T_{p} X$ for all $p \in X_{\text {reg, }}$, where \langle\rangle denotes the linear span.

Motivations. Before going on with the content of this paper, we present several reasons to study tangent spaces and arrows. The first motivation came from a key lemma due to Terracini which uses unions of tangent spaces to general points to compute the dimensions of the secant varieties of $X$ ([1, Corollary 1.10]). To compute the dimension of the linear span of finite unions of tangent spaces to $X_{\text {reg }}$ an efficient way is to use arrows. This was observed and popularized by K. E. Chandler $([9,10,11])$ who called dots

*The author was partially supported by MIUR and GNSAGA of INdAM (Italy). 
our arrows. See [3,13] for the use of arrows for a tangent space computation and hence an improvement of [18]. Recently, inspired by Terracini's lemma there was a study of the Terracini locus $\mathbb{T}_{1}(X, x)$ $([4,5])$. The main motivation came from the observation that it is easy to compute tangent spaces using a computer and that these computations help to check the identifiability of a tensor $T$ (resp. a homogeneous polynomial $f$ ), i.e. that $T$ (resp. $f$ ) is in a unique way the sum of a minimum number of rank 1 tensors (resp. powers of linear forms). For the identifiability problem, see [5, 23] and references therein.

Our set-up (Questions and Conjectures) are only for homogeneous varieties, but we hope that it is clear how to use them for many other embedded varieties. The book [21] by J. L. Landsberg concerning tensors has several chapters related to the geometry of homogeneous varieties and use of representation theory for questions related to the tensor rank. See [8] for a paper concerned (in the setting of secant varieties) to certain homogeneous varieties (the cominuscule varieties such as Grassmannians). See [22] for the use of projective geometry to get a new proof of the classification of complex simple Lie algebras.

We propose the study of the following key objects.

Key objects. Let $\rho(X)^{\prime \prime}$ be the maximal integer such that all zero-dimensional schemes $Z \subset X_{\text {reg }}$ of degree at most $\rho(X)^{\prime \prime}$ are linearly independent. Let $\rho_{w}(X)$ denote the maximal integer $t$ such that any union of $t$ arrows of $X_{\text {reg }}$ is linearly independent. Let $\mathbb{L}(X)$ denote the set of all $S \subset X_{\text {reg }}$ such that $\# S=\rho_{w}(X)+1$ and there is a union $Z \subset X$ of $\# S$ arrows with $Z_{\text {red }}=S$ and $Z$ linearly dependent. Let $\mathcal{L}(X)$ denote the set of all schemes $Z \subset X_{\text {reg }}$ which are linearly dependent and unions of $\rho_{w}(X)+1$ arrows. For any integer $x>0$ and any open subset $U \subseteq X_{\text {reg }}$ let $\mathcal{A}(U, x)$ denote the set of all unions of $x$ disjoint arrows whose reduction is contained in $U$. Set $\mathcal{A}(U):=\cup_{x>0} \mathcal{A}(U)$.

Since any degree 2 zero-dimensional subscheme of a projective space spans a line, $\rho(X)^{\prime \prime} \geq 2$. For any integer $t>0$ a union of $t$ arrows has at most degree $2 t$ and it has degree $2 t$ if and only if the reduction of these arrows is formed by $t$ distinct points. Thus $\rho_{w}(X) \geq\left\lceil\rho(X)^{\prime \prime} / 2\right\rceil$. In the definition of $\rho_{w}(X)$ we obtain the same integer if we prescribe that the arrows of $X_{\text {reg }}$ are disjoint (Lemma 2.7). In the definition of $\mathcal{L}(X)$ we get the same set if we add the condition that the arrows are disjoint (Lemma 2.7).

We see (Remark 2.1) that arrows allow one to control the Terracini loci. Arrows also define the following stratification (called the second Terracini stratification of the pair $(X, Z)$ ) induced by the second Terracini function, which we now define. Fix $x \in \mathbb{N}$ and $Z \in \mathcal{A}\left(X_{\text {reg }}, x\right)$. Set $S:=Z_{\text {red. }}$ The second Terracini function

$$
u_{X, Z}: \mathcal{A}\left(X_{\text {reg }} \backslash S\right) \rightarrow \mathbb{N}
$$

of the pair $(X, Z)$ is the function defined by the formula $v \mapsto h^{1}\left(\mathcal{I}_{Z \cup v}(1)\right)-h^{1}\left(\mathcal{I}_{Z}(1)\right)$. Note that $0 \leq u_{X, Z}(v) \leq 2$ for all $v \in \mathcal{A}\left(X_{\text {reg }} \backslash S\right)$. Note that $u_{X, Z}(v) \leq 1$ for all $v$ if $\langle Z\rangle$ is a hyperplane and $u_{X, Z}(v)=0$ for all $v$ if $\langle Z\rangle=\mathbb{P}^{r}$. Assume $\operatorname{dim}\langle Z\rangle \leq r-2$. In characteristic 0 , one has $u_{X, Z}(v)=$ 2 for a general $v$. In positive characteristic this is not always true, unless $X$ is not a strange variety $([2,6,15,16,24])$. The main justification for the study of the Terracini loci is given by Terracini's lemma ([1, Corollary 1.10]), which relates the dimension of the linear span of general unions of tangent spaces to the dimension of secant varieties of $X$. The statement of [1, Corollary 1.10] explains the main difference between characteristic zero and positive characteristic. 
Question 1.1. Assume $\operatorname{char}(\mathbb{K})=0$ and that $X \subset \mathbb{P}^{r}$ is a homogeneous projective manifold with respect to an affine algebraic group embedded by the complete linear system of a homogeneous line bundle. Assume $\operatorname{dim} X>1$. Let $d$ be the minimal degree of a smooth curve on $X$. Is $\rho_{w}(X)=\lceil d / 2\rceil$ ?

Each minimal degree $d$ curve $T \subset X$ should be embedded as a rational normal curve in its embedding.

Remark 1.2. Question 1.1 has an affirmative answer if $X \subset \mathbb{P}^{r}$ contains a line, because any degree 3 subscheme of a line is linearly dependent. Since $X$ is a homogeneous variety embedded by a homogeneous complete linear system, it is ruled by lines if it contains a line. For instance this is the case for the Segre varieties and the Grassmannians embedded by their Plücker embedding. The case of the Grassmannians with other linearly normal embeddings is covered by Proposition 3.3 and Remark 3.4.

In Theorem 1.3 and 1.5 we show that the answer to Question 1.1 is yes for the Segre-Veronese embeddings of any multiprojective space. Part (ii) of Theorem 1.5 shows that natural extensions of Question 1.1 may fail.

Theorem 1.3. Fix positive integers $k, n_{i}$ and $d_{i}, 1 \leq i \leq k$. Set $Y:=\mathbb{P}^{n_{1}} \times \cdots \times \mathbb{P}^{n_{k}}$. Let $\nu_{d_{1}, \ldots, d_{k}}: Y \rightarrow$ $\mathbb{P}^{r}, r=-1+\prod_{i=1}^{k}\left(\begin{array}{c}n_{i}+d_{i} \\ n_{i}\end{array}\right)$, denote the Segre-Veronese embedding of $Y$ with multidegree $\left(d_{1}, \ldots, d_{k}\right)$. Set $X:=\nu_{d_{1}, \ldots, d_{k}}(Y), \delta:=\min _{1 \leq i \leq k} d_{i}$ and $E:=\left\{i \in\{1, \ldots, k\} \mid d_{i}=\delta\right\}$. Then

(i) $\rho_{w}(X)=\lceil\delta / 2\rceil$ and $\delta$ is the minimal degree of a curve $T \subseteq X$.

(ii) Each curve $T \subseteq X$ with $\operatorname{deg}(T)=\delta$ is embedded as a rational normal curve in the $\delta$-dimensional linear subspace $\langle T\rangle$ and there is $i \in E$ and a curve $L \subseteq Y$ such that $T=\nu_{d_{1}, \ldots, d_{k}}(L), \pi_{j}(L)$ is a point for all $j \in\{1, \ldots, k\} \backslash\{i\}$ and $\pi_{i}(L)$ is a line.

(iii) $\rho(X)^{\prime \prime}=\delta+1$.

(iv) $\mathbb{L}(X)$ contains the set $S(T,\lceil(\delta+2) / 2\rceil), T$ a minimal degree curve, and the set $\mathcal{L}(X)$ contains the unions $Z \subset X$ of $\lceil(\delta+2) / 2)\rceil$ disjoint arrows such that $\operatorname{deg}(Z \cap T) \geq \delta+2$ for some $T$.

(v) Assume $\delta$ even. Then for every $Z \in \mathcal{L}(X)$ there is a unique degree $\delta$ curve $T \subset X$ such that $Z \subset T$.

(vi) Assume $\delta \geq 3$ and $\delta$ odd. Then for every $Z \in \mathcal{L}(X)$ there is a unique degree $\delta$ curve $T \subset X$ such that $\operatorname{deg}(Z \cap T) \geq \delta+2$ and $Z_{\text {red }} \subset T$.

Remark 1.4. Take $X$ as in Theorem 1.3. All degree $\delta$ curves $T \subset X$ are easily described (Remark 4.1) and each such $T$ is a rational normal curve of $\langle T\rangle \cong \mathbb{P}^{\delta}$. Fix any such $T$ and $Z \in \mathcal{L}(X)$. If $\delta$ is even $\operatorname{deg}(Z \cap T) \geq \delta+2$ if and only if $Z \subset T$. If $\delta$ is odd $\operatorname{deg}(Z \cap T) \geq \delta+2$ if and only if $\operatorname{deg}(Z \cap T) \geq \operatorname{deg}(Z)-1$. Since each connected component of $Z$ has degree $2, \operatorname{deg}(Z \cap T) \geq \operatorname{deg}(Z)-1$ implies $Z_{\text {red }} \subset T$. Thus (under the assumptions of (v) and (vi)), for each $S \in \mathbb{L}(X)$ there is a unique $L \subset Y$ such that $S \subset \nu_{d_{1}, \ldots, d_{k}}(L)$ and $L$ is embedded as a line by the Segre embedding $\nu_{1, \ldots, 1}$ of $Y$.

The following theorem and Example 4.2 show that part (vi) of Theorem 1.3 is not always true for $\delta=1$. 
Theorem 1.5. In the set-up of Theorem 1.3 assume $\delta=1$. Fix any $W \in \mathcal{L}(X)$ and $\operatorname{set} Z:=\nu_{d_{1}, \ldots, d_{k}}^{-1}(W)$. Assume that $Y$ is the minimal multiprojective space containing $Z$.

(i) Assume $\# E=1$, say $E=\{i\}$. For each $W \in \mathcal{L}(X)$, there is a line $L \subseteq X$ containing $W_{\text {red. If }}$ $k \geq 2$, then $\operatorname{deg}(W \cap L) \geq 3$.

(ii) Assume $\# E \geq 2$. Fix $Z \in \mathcal{L}(X)$. Call $\alpha$ the minimal degree of a curve $C \subset X$ containing the scheme $Z$. Either $\alpha=1$ or $\alpha=2, k=2, n_{1}=n_{2}=d_{1}=d_{2}=1$ and the unique curve $D \subset X$ with minimal degree containing $W$ is a conic.

In part (ii) of Theorem 1.5 we allow locally Cohen-Macaulay 1-dimensional schemes and reducible curve, i.e. we allow reducible curves and curves with multiple components (but no embedded component). The exceptional case in part (ii) occurs and when $\alpha=2$ both reducible conics and smooth conics occur (for different $W^{\prime}$ 's) as seen in Example 4.2. Thus in part (ii) of Theorem 1.5 it is essential to allow reducible curves in the definition of the integer $\alpha$. This exceptional case shows that sometimes in Question 1.1 not all elements of $\mathbb{L}(X)$ are contained in minimal degree curves.

\section{Preliminaries}

We need to use the following well-known concept of residual scheme. Let $W$ be an integral projective variety and $D$ an effective Cartier divisor of $W$. For any closed subscheme $A$ of $W$ (resp. of $D$ ) let $\mathcal{I}_{A} \subseteq \mathcal{O}_{W}$ (resp. $\mathcal{I}_{A, D} \subseteq \mathcal{O}_{D}$ ) denote its ideal sheaf. Let $Z \subset W$ be a zero-dimensional scheme. Let $Z \cap D$ denote the scheme-theoretic intersection of $Z$ and $D$, i.e. the closed subscheme of $W$ with $\mathcal{I}_{Z}+\mathcal{I}_{D}$ as its ideal sheaf. The scheme $Z \cap D$ is the closed subscheme of $D$ with as its ideal sheaf the image of $\mathcal{I}_{Z}$ by surjection $\mathcal{O}_{W} \rightarrow \mathcal{O}_{D}$. The residual scheme $\operatorname{Res}_{D}(Z)$ of $Z$ with respect to $D$ is the closed subscheme of $X$ with $\mathcal{I}_{W}: \mathcal{I}_{D}$ as its ideal sheaf. Since $\operatorname{Res}_{D}(Z) \subseteq Z, \operatorname{Res}_{D}(Z)$ is a zero-dimensional scheme. We have $\operatorname{deg}(Z)=\operatorname{deg}\left(\operatorname{Res}_{D}(Z)\right)+\operatorname{deg}(Z \cap A)$ and for each line bundle $\mathcal{L}$ on $W$ there is an exact sequence

$$
0 \rightarrow \mathcal{I}_{\operatorname{Res}_{D}(Z)} \otimes \mathcal{L}(-D) \rightarrow \mathcal{I}_{Z} \otimes \mathcal{L} \rightarrow \mathcal{I}_{D \cap Z, D} \otimes \mathcal{L}_{\mid D} \rightarrow 0
$$

For any $Z$ and $\mathcal{L}$ we will say that (1) is the residual exact sequence of $D$.

Let $U \subset \mathbb{P}^{r}$ be a zero-dimensional scheme. The linear space $\langle U\rangle$ is the intersection of all hyperplanes $H \supset U$ with the convention $\langle U\rangle=\mathbb{P}^{r}$ if there is no such hyperplane. Note that $\operatorname{dim}\langle U\rangle=\operatorname{deg}(U)-1-$ $h^{1}\left(\mathbb{P}^{r}, \mathcal{I}_{U}(1)\right)$.

Remark 2.1. For any $p \in X_{\text {reg }}$ the linear space $T_{p} X$ is spanned by $\operatorname{dim} X$ arrows with $\{p\}$ as their reduction. Thus for any finite set $S \subset X_{\text {reg }}, S \neq \emptyset$, the linear space $\langle(2 S, X)\rangle=\left\langle\cup_{p \in S} T_{p} X\right\rangle$ is the linear subspace of $\mathbb{P}^{r}$ spanned by all arrows with as reduction one of the points of $S$.

Remark 2.2. The set $\mathcal{A}\left(X_{\text {reg }}\right)$ is a smooth and irreducible quasi-projective variety and $\operatorname{dim} \mathcal{A}\left(X_{\text {reg }}\right)=$ $2 n-1$, where $n:=\operatorname{dim} X$. For any positive integers $x$ the set $\mathcal{A}\left(X_{\text {reg }}, x\right)$ is a quasi-projective smooth and irreducible variety of dimension $x n(n-1)$.

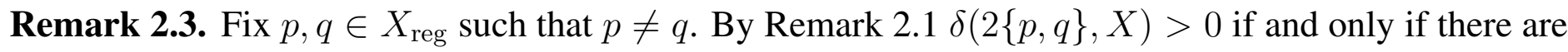
arrows $v, w \subset X_{\text {reg }}$ such that $v_{\text {red }}=\{p\}, w_{\text {red }}=\{q\}$ and $\operatorname{dim}\langle v \cup w\rangle \leq 2$.

Remark 2.4. Since $\mathcal{O}_{X}(1)$ is very ample, $t_{X, \emptyset}$ is the constant function $t_{X, \emptyset}(u)=\operatorname{dim} X+1$ for all $u \in X_{\text {reg. }}$. Remark 2.1 gives the existence of a set $S \subset X_{\text {reg }}$ such that $t_{X, S}$ is not a constant function if 
there is an integral curve $T \subset X$ such that $T \cap X_{\text {reg }} \neq \emptyset$ and $\operatorname{dim}\langle T\rangle \leq\lfloor(r+1) /(\operatorname{dim} X+1)\rfloor-1$. Easy examples show that the existence of $T$ is not a necessary condition (Example 2.5 and Lemma 2.8).

Example 2.5. Take $\operatorname{dim} X=1$. In this case no proper subspace of $\mathbb{P}^{r}$ contains a subcurve of $X$, because $X$ is assumed to be non-degenerate. Take $r=3$. There are some $X$ such that there are arrows $v, w \subset X_{\text {reg }}$ such that $v_{\text {red }} \neq w_{\text {red }}$ and $\operatorname{dim}\langle v \cup w\rangle \leq 2$. Indeed, this occurs quite often, since it is sufficient to take $X$ such that there is $p \in X_{\text {reg }}$ with $T_{p} X \cap\left(X_{\text {reg }} \backslash\{p\}\right) \neq \emptyset$. Take for instance a general canonically embedded curve of genus 4 and degree 6 . In this example there are only finitely many such points $p$. When $\operatorname{dim} X=1$ an arrow is uniquely determined by its reduction. In this example there are only finitely many $p$ 's doing the job. Of course, for $r=2$ and $X$ not a smooth conic we may take as $p$ a

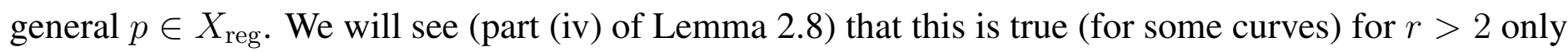
in positive characteristic.

Lemma 2.6. Let $V_{1}, \ldots, V_{s}$ be non-empty linear subspaces of $\mathbb{P}^{r}$. Assume that $V_{1}, \ldots, V_{s}$ are linearly dependent. Then there are $o_{i} \in V_{i}, 1 \leq i \leq s$, such that $\left\{o_{1}, \ldots, o_{s}\right\}$ is linearly dependent.

Proof. Since $V_{1}, \ldots, V_{s}$ are linearly dependent, $s \geq 2$. Using induction on $s$ we reduce to the case in which $V_{1}, \ldots, V_{s-1}$ are linearly independent. Since $V_{1}, \ldots, V_{s}$ are linearly dependent, $\left\langle V_{1} \cup \cdots \cup V_{s-1}\right\rangle \cap$ $V_{s} \neq \emptyset$. Fix $q \in\left\langle V_{1} \cup \cdots \cup V_{s-1}\right\rangle \cap V_{s}$. Take $o_{i} \in V_{i}, 1 \leq i \leq s-1$, such that $q \in\left\langle\left\{o_{1}, \ldots, o_{s-1}\right\}\right\rangle$. Set $o_{s}:=q$.

Lemma 2.7. Let $X \subset \mathbb{P}^{r}$ be an integral and non-degenerate variety.

(a) Every $Z \in \mathcal{L}(X)$ has degree $2 \rho_{w}(X)+2$.

(b) $\rho_{w}(X)+1$ is the minimal integer $x$ such that there is $S \in S\left(X_{\mathrm{reg}}, x\right)$ with the property that the linear spaces $T_{p} X, p \in S$, are linearly dependent.

Proof. Part (a) means that each $Z \in \mathcal{L}(X)$ is the union of $\rho_{w}(X)$ disjoint arrows. Both parts are obvious if $n=1$. Thus we may assume $n>1$. We first prove part (b). Let $s$ be the minimal integer such that that there is $S \in S\left(X_{\mathrm{reg}}, s\right)$ with the property that the linear spaces $T_{p} X, p \in S$, are linearly dependent. Since every arrow with $p \in X_{\text {reg }}$ as its reduction is contained in $T_{p} X, s \leq \rho_{w}(X)+1$. By Lemma 2.6 there are $o_{p} \in T_{p} X, p \in S$, such that $\cup_{p \in S}\left\{o_{p}\right\}$ is linearly dependent. If $o_{p} \neq p$ let $v_{p}$ be the arrow with $p$ as its reduction and with $\left\langle\left\{p, o_{p}\right\}\right\rangle$ as its linear span. If $o_{p}=p$ let $v_{p}$ be an arbitrary arrow with $p$ as its reduction. Since $\cup_{p \in S} v_{p}$ is linearly dependent, we get $s \geq \rho_{w}(X)+1$ concluding the proof of part (b).

Take $Z \in \mathcal{L}(X)$. Part (b) implies that $\# Z_{\text {red }} \geq \rho_{w}(X)+1$. Thus $Z$ is the union of $\rho_{w}(X)+1$ disjoint arrows.

For any linear subspace $V \subset \mathbb{P}^{r}$ let $\ell_{V}: \mathbb{P}^{r} \backslash V \rightarrow \mathbb{P}^{r-s-1}, s:=\operatorname{dim} V$, denote the linear projection from $V$. For any integral $X \subset \mathbb{P}^{r}$ such that $X \nsubseteq V$ let $X_{V} \subseteq \mathbb{P}^{r-s-1}$ denote the closure of $\ell_{V}(X \backslash X \cap V)$ in $\mathbb{P}^{r-s-1}$.

Lemma 2.8. Let $X \subset \mathbb{P}^{r}$ be an integral and non-degenerate variety. Fix $S \in S\left(X_{\mathrm{reg}}, x\right)$ and a general $A \in S\left(X_{\mathrm{reg}}, x\right)$. Set $n:=\operatorname{dim} X, m:=\operatorname{dim}\langle(2 S, X)\rangle$ and $\mu:=\operatorname{dim}\langle(2 A, X)\rangle$.

(i) $m \leq \mu$. 
(ii) Assume $r \geq \mu+n+1$ (resp. $r \geq m+n+1$ ). Then: $t_{X, S}(u)>\delta(2 S, X)$ (resp. $\left.t_{X, S}(u)>\delta(2 S, X)\right)$ for all $u \in X_{\mathrm{reg}} \backslash S$ (resp. all $u \in X_{\mathrm{reg}} \backslash A$ ) if and only if this is true for a general $u \in X_{\mathrm{reg}}$.

(iii) Assume $r \geq m+n+1$. $t_{X, S}(u)>\delta(2 S, X)$ (resp. $t_{X, S}(u)>\delta(2 S, X)$ for a general $\left.u \in X_{\mathrm{reg}}\right)$ if and only if $T_{u} X \cap\langle(2 S, X)\rangle \neq \emptyset$ (resp. $T_{u} X \cap\langle(2 S, X)\rangle \neq \emptyset$ for a general $\left.u \in X_{\mathrm{reg}}\right)$.

(iv) Assume $r \geq m+n+1$ and $\operatorname{char}(\mathbb{K})=0 . t_{X, S}(u)>\delta(2 S, X)$ for a general $u \in X_{\mathrm{reg}}$ if and only $\operatorname{dim} X_{V}<n$, where $V:=\langle(2 S, X)\rangle$.

Proof. Parts (i) and (ii) follow from the semicontinuity theorem for cohomology. Part (iii) follows from the definition of the Terracini function $t_{X, S}$ and of the defect $\delta(2(--), X)$, while part (iv) follows from the definition of $\ell_{V}$ and $X_{V}$, the assumption that $X$ is non-degenerate and the fact that in characteristic zero the dimension of the image of a rational map $f$ with as its domain an irreducible variety is the generic rank of the differential of $f$.

Remark 2.9. Let $X \subset \mathbb{P}^{r}$ be an integral non-degenerate variety and $C \subset X$ an integral curve. Set $z:=\operatorname{dim}\langle C\rangle$. Every zero-dimensional scheme $Z \subset C$ such that $\operatorname{deg}(Z) \geq m+2$ is linear dependent.

Remark 2.10. Assume $\operatorname{char}(\mathbb{K})=0$. By [12] for any non-degenerate $X \subset \mathbb{P}^{r}$ and any positive integer $x \operatorname{dim}\langle Z\rangle=\min \{r, 2 x-1\}$ for a general $Z \in \mathcal{A}\left(X_{\text {reg }}, x\right)$. Thus $u_{X, Z}(u)=\min \{2, r+1-2 x\}$ for a general $Z \in \mathcal{A}\left(X_{\text {reg }}, x\right)$ and a general $u \in \mathcal{A}\left(X_{\text {reg }} \backslash Z_{\text {red }}\right)$. Theorem 1.5 gives the function $u_{X, v}$ when $X$ is a Segre variety and $v$ is any any arrows $v \subset X$.

Remark 2.11. A theorem of Luiss says that there is a unique smooth strange curve (if we exclude the lines): a smooth plane conic in characteristic 2 (see [14, IV.3.9] or [20, Proposition 3]).

\section{Veronese embeddings}

Before proving Theorem 1.3 we prove the case $k=1$ of it, i.e. the case of a Veronese embedding of a projective space, to prove our result on the Grassmannians (Remark 3.4) and to start the proof of Theorem 1.3 by induction on $k$.

Remark 3.1. Fix positive integers $n$ and $d$. Let $\nu_{d}: \mathbb{P}^{n} \rightarrow \mathbb{P}^{r}, r=-1+\left(\begin{array}{c}n+d \\ n\end{array}\right)$, denote the order $d$ Veronese embedding of $\mathbb{P}^{n}$. Let $A \subset \mathbb{P}^{n}$ be a zero-dimensional scheme such that $\operatorname{deg}(A) \leq d+2$ and $\nu_{d}(A)$ is linearly dependent. Then $\operatorname{deg}(A)=d+2$ and there is a line $L \subseteq \mathbb{P}^{n}$ such that $A \subset L$ ([7, Lemma 34]).

Proposition 3.2. Fix positive integers $n$ and $d$. Let $\nu_{d}: \mathbb{P}^{n} \rightarrow \mathbb{P}^{r}, r=-1+\left(\begin{array}{c}n+d \\ n\end{array}\right)$, denote the order $d$ Veronese embedding of $\mathbb{P}^{n}$. Set $X:=\nu_{d}\left(\mathbb{P}^{n}\right)$.

(i) $d$ is the minimal degree of an integral curve $T \subseteq X$ and all such curves are of the form $\nu_{d}(L)$ for some line $L \subseteq \mathbb{P}^{n}$.

(ii) $\rho_{w}(X)=\lceil d / 2\rceil$ and for each $S \in \mathbb{L}(X)$ there is a unique line $L \subseteq \mathbb{P}^{n}$ such that $S \subset \nu_{d}(L)$.

(iii) Assume either $n=1$ or $d$ even. Then $Z \in \mathcal{L}(X)$ if and only if $\operatorname{deg}(Z)=d+2$ and there is a line $L \subseteq \mathbb{P}^{n}$ such that $Z \subset \nu_{d}(L)$.

(iv) Assume $n>1$ and $d$ odd and take any $Z \in \mathcal{L}(X)$. Then there is a unique line $L \subset \mathbb{P}^{n}$ such that $d+2 \leq \operatorname{deg}\left(Z \cap \nu_{d}(L)\right) \leq d+3=\operatorname{deg}(Z)$. 
Proof. First assume $n=1$. In this case $\operatorname{deg}(X)=d$ and $X$ contains no proper projective subcurve. Hence in this case for each zero-dimensional scheme $Z \subset X$ there is a unique line $L \subseteq \mathbb{P}^{n}$ (just $L=\mathbb{P}^{1}$ ) with $Z \subset \nu_{d}(L)$. Thus in this case some of the assertions are trivially true. In this case $r=d$ and hence each zero-dimensional scheme $Z \subset X$ with $\operatorname{deg}(Z) \geq d+2$ is linearly dependent. For each zerodimensional scheme $A \subset \mathbb{P}^{1}, h^{1}\left(\mathbb{P}^{1}, \mathcal{I}_{A}(d)\right)>0$ if and only if $\operatorname{deg}(A) \geq d+2$. Thus $\rho(X)^{\prime \prime}=d+1$. Thus $\rho_{w}(X) \geq\lceil d / 2\rceil$. Remark 2.9 gives $\rho_{w}(X) \leq\lceil d / 2\rceil$.

From now on we assume $n>1$. For each integral curve $T \subset X, D:=\nu_{d}^{-1}(T)$ is an integral curve, $T=\nu_{d}(D)$ and $\operatorname{deg}(T)=d \operatorname{deg}(D)$. Thus the description of minimal degree curves is obvious. Taking $\nu_{d}(L)$ with $L$ a line we also get $\rho(X)^{\prime \prime} \leq d+1$ and $\rho_{w}(X)^{\prime \prime} \leq\lceil d / 2\rceil$. Take a zero-dimensional scheme $Z \subset X$ such that $z:=\operatorname{deg}(Z) \leq 2 d+1$ and $\operatorname{dim}\langle Z\rangle \leq z-2$, where \langle\rangle denote the linear span. Set $A:=\nu_{d}^{-1}(Z)$. Since $\operatorname{dim}\langle Z\rangle \leq z-2, h^{1}\left(\mathbb{P}^{n}, \mathcal{I}_{A}(d)\right)>0$. Since $z \leq 2 d+1$, there is a line $L \subset \mathbb{P}^{n}$ such that $\operatorname{deg}(Z \cap L) \geq d+2$ ([7, Lemma 34]), concluding the proof of (iii) and (iv).

The next result shows that Proposition 3.2 is useful for Veronese embeddings of subvarieties of a projective space.

Proposition 3.3. Let $Y \subseteq \mathbb{P}^{m}$ be an integral and non-degenerate variety. Let $\nu_{d}: \mathbb{P}^{m} \rightarrow \mathbb{P}^{N}, N=$ $-1+\left(\begin{array}{c}m+d \\ d\end{array}\right)$, be the order $d$ Veronese embedding of $\mathbb{P}^{m}$. Set $X:=\nu_{d}(Y)$ and $\mathbb{P}^{r}:=\langle X\rangle$.

(a) If there is a line $L \subset \mathbb{P}^{m}$ such that either $L \subset Y$ and $L \cap Y_{\text {reg }} \neq \emptyset$ or $\operatorname{deg}\left(L \cap Y_{\text {reg }}\right) \geq d+2$, then $\rho(X)^{\prime \prime}=d+1$, while $\rho(X)^{\prime \prime} \geq d+2$ if there is no such line.

(b) $\rho_{w}(X)>\lceil d / 2\rceil$ if there is no line $L \subset \mathbb{P}^{m}$ containing at least $\lceil d / 2\rceil$ points of $Y_{\text {reg. }}$.

Proof. For any curve $T \subseteq \mathbb{P}^{m}, \operatorname{deg}\left(\nu_{d}(T)\right)=d \operatorname{deg}(T)$. Thus each curve $C \subset \nu_{d}\left(\mathbb{P}^{m}\right)$ has degree divisible by $d, d$ is the minimal degree of a curve contained in $\nu_{d}\left(\mathbb{P}^{m}\right)$ and all minimal degree curves of $\nu_{d}\left(\mathbb{P}^{m}\right)$ are rational normal curves in their linear span (here we use that for each line $L \subset \mathbb{P}^{m}$ the restriction map $H^{0}\left(\mathcal{O}_{\mathbb{P} m}(d)\right) \rightarrow H^{0}\left(\mathcal{O}_{L}(d)\right)$ is surjective). Parts (a) and (b) follows from the inequalities $\rho(X)^{\prime \prime} \geq \rho\left(\nu_{d}\left(\mathbb{P}^{m}\right)\right)^{\prime \prime}$ and $\rho_{w}(X) \geq \rho_{w}\left(\nu_{d}\left(\mathbb{P}^{m}\right)\right)$ and parts (iii) and (iv) of Proposition 3.2.

Remark 3.4. Take as $Y$ in Proposition 3.3 a Grassmannian $G(a, b)$ with its Plücker embedding. Since $\mathcal{O}_{G(a, b)}(1)$ generates $\operatorname{Pic}(G(a, b))$ and $G(a, b)$ contains lines, Proposition 3.3 computes $\rho(X)^{\prime \prime}$ and $\rho_{w}(X)$ for each linearly normal embedding $X$ of a Grassmannian.

\section{Proofs of Theorems 1.3 and 1.5}

Set $Y:=\mathbb{P}^{n_{1}} \times \cdots \times \mathbb{P}^{n_{k}}$. Let $\pi_{i}: Y \rightarrow \mathbb{P}^{n_{i}}$ denote the projection onto the $i$-th factor of $Y$. For $i=1, \ldots, k$ let $\varepsilon_{i}$ (resp. $\hat{\varepsilon}_{i}$ ) be the multi-index $\left(a_{1}, \ldots, a_{k}\right) \in \mathbb{N}^{k}$ such that $a_{i}=1$ and $a_{j}=0$ for all $j \neq 1$ (resp. $a_{i}=0$ and $a_{j}=1$ for all $j \neq i$ ). For any integral curve $D \subset Y$ we define the multidegree $\left(a_{1}, \ldots, a_{k}\right) \in \mathbb{N}^{k}$ of $D$ in the following way. If $\pi_{i}(D)$ is a point set $a_{i}:=0$. If $\pi_{i}(D)$ is a curve set $a_{i}:=\operatorname{deg}\left(\pi_{i \mid D}\right) \operatorname{deg}\left(\pi_{i}(D)\right)$, with the convention $\operatorname{deg}\left(\pi_{i}(D)\right)=1$ if $n_{i}=1$. Since $D \neq \emptyset, a_{i}>0$ for at least one index $i$.

Remark 4.1. Let $D \subset Y$ be an integral curve of multidegree $\left(a_{1}, \ldots, a_{k}\right)$. As in Theorem 1.3 set $\delta:=\min \left\{d_{1}, \ldots, d_{k}\right\}$ and let $E$ be the set of all $i \in\{1, \ldots, k\}$ such that $d_{i}=\delta$. Note that $\nu_{d_{1}, \ldots, d_{k}}(D)$ has degree $a_{1} d_{1}+\cdots+a_{k} d_{k}$. Thus $\operatorname{deg}\left(\nu_{d_{1}, \ldots, d_{k}}(D)\right) \geq \delta$ and equality holds if and only if there is 
$i \in E$ such that $a_{i}=1$ and $a_{j}=0$ for all $j \in\{1, \ldots, k\} \backslash\{i\}$. Fix $i \in E$. All irreducible curves with multidegree $\varepsilon_{i}$ are obtained in the following way. For all $j \in\{1, \ldots, k\} \backslash\{i\}$ fix $o_{j} \in \mathbb{P}^{n_{j}}$. If $n_{i}=1$ let $f_{i}: \mathbb{P}^{1} \rightarrow \mathbb{P}^{n_{i}}$ be an isomorphism. If $n_{i}>1$, then $f_{i}: \mathbb{P}^{1} \rightarrow \mathbb{P}^{n_{i}}$ be an embedding with $f_{i}\left(\mathbb{P}^{1}\right)$ a line. Let $f: \mathbb{P}^{1} \rightarrow Y$ be the only morphism such that $\pi_{h} \circ f=f_{h}$ for all $h \in\{1, \ldots, k\}$. Since $f_{i}$ is an embedding, $f$ is an embedding. Thus $f\left(\mathbb{P}^{1}\right) \cong \mathbb{P}^{1}$. The curve $f\left(\mathbb{P}^{1}\right)$ has multidegree $\varepsilon_{i}$ and all curves with multidegree $\varepsilon_{i}$ are obtained in this way for some choice of the points $o_{j} \in \mathbb{P}^{n_{j}}, j \neq i$, and, if $n_{i}>1$, a line of $\mathbb{P}^{n_{i}}$.

Let $D \subset Y$ be any curve such that $\operatorname{deg}\left(\nu_{d_{1}, \ldots, d_{k}}(D)\right)=\delta$. Note that the description just given shows that $h^{1}\left(D, \mathcal{I}_{D}\left(b_{1}, \ldots, b_{k}\right)\right)=0$ for all $\left(b_{1}, \ldots, b_{k}\right) \in \mathbb{N}^{k}$. Thus $\nu_{d_{1}, \ldots, d_{k}}(D)$ is a degree $\delta$ rational normal curve in its linear span.

Example 4.2. Take $Y=\mathbb{P}^{1} \times \mathbb{P}^{1}$ and $d_{1}=d_{2}=1$. Since $r=3$ in this case, $h^{1}\left(Y, \mathcal{I}_{Z}(1)\right)>0$ for a degree 4 zero-dimensional scheme if and only if $\nu_{1,1}(Z)$ is contained in a plane. If $\operatorname{deg}(H \cap Z) \leq 2$ for all multiprojective spaces $Y^{\prime} \subsetneq Y$, then $Z$ is as in one of the two cases:

(a) Fix $\left(o_{1}, o_{2}\right) \in \mathbb{P}^{1} \times \mathbb{P}^{1}$ and set $L:=\pi_{1}^{-1}\left(o_{1}\right)$ and $R:=\pi_{2}^{-1}\left(o_{2}\right)$. Take $a \in L \backslash L \cap R$ and $b \in R \backslash R \cap L$. Let $v \subset L$ be the only arrow with $v_{\text {red }}=\{a\}$ and let $w \subset R$ be the only arrow with $w_{\text {red }}=\{b\}$.

(b) Take an irreducible $D \in\left|\mathcal{O}_{Y}(1,1)\right|$. Since $\nu_{1,1}(D)$ is a smooth conic, $D$ is smooth. Take $a, b \in D$ such that $a \neq b$ and take as $v$ (resp. $w$ ) the only degree two connected zero-dimensional subscheme of $D$ with $\{a\}$ (resp. $\{b\}$ ) as its reduction.

To prove that all cases are as in (a) or (b) note that (since $h^{0}\left(\mathcal{O}_{Y}(1,1)\right)=4$ and $h^{1}\left(Y, \mathcal{I}_{Z}(1,1)\right)>0$ by assumption) $\left|\mathcal{I}_{Z}(1,1)\right| \neq \emptyset$. Take $T \in\left|\mathcal{I}_{Z}(1,1)\right|$. If $T$ is irreducible, then $Z$ is as in case (b) with $D=T$. Assume $T$ reducible, say $T=H_{1} \cup H_{2}$. If $\operatorname{deg}\left(Z \cap H_{i}\right) \geq 3$ for some $i$, then we take $H_{i}$ as the linee with $\operatorname{deg}\left(H_{i} \cap Z\right) \geq 3$. If $\operatorname{deg}\left(Z \cap H_{1}\right) \leq 2$ and $\operatorname{deg}\left(Z \cap H_{2}\right) \leq 2$, then $\operatorname{deg}\left(Z \cap H_{1}\right)=\operatorname{deg}\left(Z \cap H_{2}\right)=2$, $Z \cap H_{1} \cap H_{2}=\emptyset$ and $Z$ is as in case (a).

Lemma 4.3. Take the assumptions of Theorem 1.3. Then $\rho(X)^{\prime \prime}=\delta+1$.

Proof. By Proposition 3.2 the lemma is true if $k=1$. Thus we may assume $k>1$. Hence $\operatorname{dim} Y>1$ and we may use induction on the integer $\operatorname{dim} Y$. Since $X$ contains a degree $\delta$ curve (Remark 4.1), $\rho(X)^{\prime \prime} \leq \delta+1$ (Remark 2.9). Since $\mathcal{O}_{X}(1)$ is very ample, $\rho(X)^{\prime \prime} \geq 2$. Thus the lemma is true if $\delta=1$. Thus we may assume $\delta \geq 2$ and that the lemma is true for all Segre-Veronese embeddings of any multiprojective space with dimension $\leq \operatorname{dim} Y$ and with curves of degree $<\delta$. Assume $y:=\rho(X)^{\prime \prime} \leq \delta$ and take a zero-dimension scheme $Z \subset X$ such that $\operatorname{deg}(Z)=y+1$ and $\operatorname{dim}\langle Z\rangle \leq y-2$, i.e. such that $h^{1}\left(Y, \mathcal{I}_{A}\left(d_{1}, \ldots, d_{k}\right)\right)>0$, where $A:=\nu_{d_{1}, \ldots, d_{k}}^{-1}(Z)$. Up to a permutation of the factors of $Y$ we may assume $d_{1}=\delta$. Fix $H \in\left|\mathcal{O}_{Y}\left(\varepsilon_{1}\right)\right|$ such that $A \cap H \neq \emptyset$. The residual scheme $\operatorname{Res}_{H}(A)$ is contained in $A, \operatorname{deg}(A)=\operatorname{deg}(A \cap H)+\operatorname{deg}\left(\operatorname{Res}_{H}(Z)\right)$. Since $H$ is a multiprojective space and $\operatorname{dim} H<\operatorname{dim} Y$, the lemma is true for $H$. Consider the residual exact sequence of $H$ :

$$
\begin{aligned}
0 \rightarrow & \mathcal{I}_{\operatorname{Res}_{H}(A)}\left(d_{1}-1, d_{2}, \ldots, d_{k}\right) \rightarrow \mathcal{I}_{A}\left(d_{1}, d_{2}, \ldots, d_{k}\right) \rightarrow \\
& \mathcal{I}_{H \cap A, H}\left(d_{1}, \ldots, d_{k}\right) \rightarrow 0
\end{aligned}
$$

The embedding $\nu_{d_{1}, \ldots, d_{k} \mid H}$ induces the Segre-Veronese embedding of $H$ with multidegree $\left(d_{2}, \ldots, d_{k}\right)$ (case $n_{1}=1$ ) or multidegree $\left(d_{1}, \ldots, d_{k}\right)$ (case $n_{1}>1$ ). Since $d_{i} \geq \delta$ for all $i$ and $\operatorname{deg}(H \cap A) \leq$ 
$\operatorname{deg}(A) \leq \delta+1, h^{1}\left(H, \mathcal{I}_{A \cap H, H}\left(d_{1}, \ldots, d_{k}\right)\right)=0$ by the two inductive assumptions. Thus (2) gives $h^{1}\left(Y, \mathcal{I}_{\operatorname{Res}_{H}(A)}\left(\delta-1, d_{2}, \ldots, d_{k}\right)\right)>0$. Since $d_{i} \geq \delta$ for all $i$ and $\operatorname{deg}\left(\operatorname{Res}_{H}(A)\right)=\operatorname{deg}(A)-\operatorname{deg}(A \cap$ $H) \leq \operatorname{deg}(A)-1 \leq \delta$, the inductive assumption gives $h^{1}\left(Y, \mathcal{I}_{\operatorname{Res}_{H}(A)}\left(\delta-1, d_{2}, \ldots, d_{k}\right)\right)=0$, a contradiction.

Lemma 4.4. Take the assumptions of Theorem 1.3. Let $Z \subset X$ be a zero-dimensional scheme such that $\operatorname{deg}(Z)=\delta+2$ and $h^{1}\left(\mathcal{I}_{Z}(1)\right)>0$. Then there is a unique degree $\delta$ curve $T \subset X$ such that $Z \subset T$.

Proof. The uniqueness of $T$ is obvious, because the description of Remark 4.1 shows that if two minimal degree curves meet, then their scheme-theoretic intersection has degree 1 . The existence of $T$ is proved in the following way. Set $A:=\nu_{d_{1}, \ldots, d_{k}}^{-1}(Z)$. With no loss of generality we may assume that $Y$ is the minimal multiprojective space containing $Z$. First assume $\delta=1$. In this case $\operatorname{deg}(Z)=3$ and $\langle Z\rangle$ is a line, $L$. Since any Segre variety is scheme-theoretically cut out by quadrics, $L \subset X$. Thus $L$ proves the lemma in this case. Hence we may assume $\delta \geq 2$ and use induction on $\delta$.

The case $k=1$ is Remark 3.1. Now assume $k>1$. By the minimality of $Y$ we need to find a contradiction. Set $A_{0}:=A$. Take $H_{1} \in\left|\mathcal{O}_{Y}\left(\varepsilon_{1}\right)\right|$ such that $e_{1}:=\operatorname{deg}\left(A_{0} \cap H_{1}\right)$ is maximal and set $A_{1}:=\operatorname{Res}_{H_{1}}\left(A_{0}\right)$. Assume defined the zero-dimensional schemes $A_{i}$, the non-negative integers $e_{i}$ and $H_{i} \in\left|\mathcal{O}_{Y}\left(\varepsilon_{1}\right)\right|$ for all $i \leq j$ for some $j \in\left\{1, \ldots, d_{1}-1\right\}$. We take any $H_{j+1} \in\left|\mathcal{O}_{Y}\left(\varepsilon_{1}\right)\right|$ such that $e_{j+1}:=$ $\operatorname{deg}\left(A_{j} \cap H_{j+1}\right)$ is maximal and set $A_{j+1}:=\operatorname{Res}_{H_{j+1}}\left(A_{j}\right)$. Note that $\operatorname{deg}\left(A_{j}\right)=\operatorname{deg}\left(A_{j+1}\right)+e_{j+1}$. The maximality of all integers $e_{i}$ gives that the sequence $\left\{e_{i}\right\}_{1 \leq i \leq d_{1}}$ is non-increasing and that if $A_{i} \neq \emptyset$, then $e_{i+1}>0$. For all $i=1, \ldots, d_{1}$ there is the following exact sequence (the residual exact sequence of $H_{i}$ ):

$$
\begin{aligned}
& 0 \rightarrow \mathcal{I}_{A_{i}}\left(d_{1}-i, d_{2}, \ldots, d_{k}\right) \rightarrow \mathcal{I}_{A_{i-1}}\left(d_{1}-i+1, d_{2}, \ldots, d_{k}\right) \rightarrow \\
& \mathcal{I}_{A_{i-1} \cap H_{i}, H_{i}}\left(d_{1}-i+1, d_{2}, \ldots, d_{k}\right) \rightarrow 0
\end{aligned}
$$

(a) In this step we assume the existence of some $i \in\left\{1, \ldots, d_{1}\right\}$ such that $h^{1}\left(H_{i}, \mathcal{I}_{A_{i-1} \cap H_{i}, H_{i}}\left(d_{1}-i+\right.\right.$ $\left.\left.1, d_{2}, \ldots, d_{k}\right)\right)>0$ and we call $g$ the first such an integer $i$. By Lemma 4.3 applied to the multiprojective space $H_{g}, e_{g} \geq 2+\min \left\{d_{1}-g+1, \delta\right\}$. Since the sequence $\left\{e_{i}\right\}_{1 \leq i \leq d_{1}}$ is non-increasing, we get $\delta+2 \geq 2 g+g \min \left\{d_{1}-g+1, \delta\right\}+\operatorname{deg}\left(A_{g}\right)$. Since $\delta \leq d_{1}$, we get $g=1, d_{1}=\delta$ and $A_{1}=\emptyset$, contradicting the assumption that $Y$ is the minimal multiprojective space containing $A$.

(b) Assume $h^{1}\left(H, \mathcal{I}_{A_{i} \cap H_{i}, H_{i}}\left(d_{1}-i+1, d_{2}, \ldots, d_{k}\right)\right)=0$ for all $i=1, \ldots, d_{1}$. Since $h^{1}\left(\mathcal{I}_{A}\left(d_{1}, \ldots, d_{k}\right)\right)$ $>0$, using all exact sequences (3) for $i=1, \ldots, d_{1}$ we get $h^{1}\left(\mathcal{I}_{A_{d_{1}}}\left(0, d_{2}, \ldots, d_{k}\right)\right)>0$. Since $\mathcal{O}_{Y}\left(0, d_{2}, \ldots, d_{k}\right)$ is globally generated, $\operatorname{deg}\left(A_{d_{1}}\right) \geq 2$. Hence $e_{1} \geq \cdots \geq e_{d_{1}}>0$, Since $\operatorname{deg}\left(A_{1}\right)+e_{1}+$ $\cdots+e_{d_{1}}=\delta+2$, we get $d_{1}=\delta, e_{i}=1$ for all $i$ and $\operatorname{deg}\left(A_{d_{1}}\right)=2$. Since $h^{1}\left(\mathcal{I}_{A_{d_{1}}}\left(0, d_{2}, \ldots, d_{k}\right)\right)>0$ and $\operatorname{deg}\left(A_{d_{1}}\right)=2, \pi_{1}\left(A_{d_{1}}\right)$ is a single point, as a scheme. The maximality of the integer $e_{1}$ gives $e_{1} \geq 2$, a contradiction.

Lemma 4.5. Assume $k \geq 2$. Let $B \subset Y$ be a zero-dimensional scheme such that $\operatorname{deg}(B) \leq 3$ and $h^{1}\left(\mathcal{I}_{B}\left(0, d_{2}, \ldots, d_{k}\right)\right)>0$. Then either there is a scheme $U \subseteq B$ such that $\operatorname{deg}(U)=2$ and $\pi_{1}(U)$ is scheme-theoretically a point or $\operatorname{deg}(B)=3$ and there is $i \in\{2, \ldots, k\}$ such that $d_{i}=1, \pi_{j}(B)$ is (scheme-theoretically) a point and for each $j \in\{2, \ldots, k\} \backslash\{i\}, \pi_{i \mid B}$ is an embedding and $\pi_{i}(B)$ is contained in a line.

Proof. Set $Y_{1}:=\prod_{i \geq 2} \mathbb{P}^{n_{i}}$ and let $\eta_{1}: Y \rightarrow Y_{1}$ denote the projection onto the last $k-1$ factors of $Y$. The first possibility occurs if and only if $\eta_{1 \mid B}$ is not an embedding. Thus we may assume that $\eta_{1 \mid B}$ 
is an embedding. Thus $h^{1}\left(\mathcal{I}_{B}\left(0, d_{2}, \ldots, d_{k}\right)\right)=h^{1}\left(Y_{1}, \mathcal{I}_{\eta_{1}(B), Y_{1}}\left(d_{2}, \ldots, d_{k}\right)\right)$. Apply Remark 4.1 and Lemmas 4.3 and 4.4 to $Y_{1}$.

Proof of Theorem 1.5. Set $S:=Z_{\text {red. }}$ Lemma 2.7 gives $\# S=2$ and $\operatorname{deg}(Z)=4$. If $k=1$, then $\# E=1, X=\mathbb{P}^{r}$ and part (i) of the theorem just say that any two points of a projective space are collinear. From now on we assume $k \geq 2$.

(a) Assume $k>\# E$. Permuting if necessary the factors of $Y$ we may assume $d_{k} \geq 2$. Thus there are $H, M \in\left|\mathcal{O}_{Y}\left(\varepsilon_{k}\right)\right|$ such that $S \subset H \cup M$, with, say, $\operatorname{deg}(Z \cap H) \geq \operatorname{deg}(Z \cap M)$. Since $Y$ is the minimal multiprojective space containing $Z, \operatorname{deg}(Z \cap H) \leq 3$. Thus $2 \leq \operatorname{deg}(Z \cap H) \leq 3$. In both cases $\operatorname{deg}\left(\operatorname{Res}_{H}(Z)\right) \leq 2$. Since $\mathcal{O}_{Y}\left(d_{1}, \ldots, d_{k-1}, d_{k}-1\right)$ is very ample and $\operatorname{deg}\left(\operatorname{Res}_{H}(Z)\right) \leq 2$, we have $h^{1}\left(Y, \mathcal{I}_{\operatorname{Res}_{H}(Z)}\left(d_{1}, \ldots, d_{k-1}, d_{k}-1\right)\right)=0$. Since $h^{1}\left(Y, \mathcal{I}_{Y}\left(d_{1}, \ldots, d_{k}\right)\right)>0$, the residual exact sequence of $H$ gives $h^{1}\left(H, \mathcal{I}_{Z \cap H, H}\left(d_{1}, \ldots, d_{k}\right)\right)>0$. Since the line bundle $\mathcal{O}_{H}\left(d_{1}, \ldots, d_{k-1}, d_{k}\right)$ is very ample, we get $\operatorname{deg}(H \cap Z)=3$.

Since $h^{1}\left(H, \mathcal{I}_{Z \cap H, H}\left(d_{1}, \ldots, d_{k}\right)\right)>0, \nu_{d_{1}, \ldots, d_{k}}(Z)$ spans a line. This line corresponds to some $i \in E$ and an integral curve with multidegree $\varepsilon_{i}$ containing $Z \cap H$.

(b) Assume $\# E=k$. Thus $d_{i}=1$ for all $i$, i.e. $X$ is a Segre variety with $k$ factors.

Assume for the moment the existence of $i \in\{1, \ldots, k\}$, such that $\# \pi_{i}(S)=1$. Thus there is $H^{\prime} \in$ $\left|\mathcal{I}_{S}\left(\varepsilon_{i}\right)\right|$. Since $H^{\prime}$ is a multiprojective space and $Y$ is the minimal multiprojective space containing $Z$, $\operatorname{deg}\left(Z \cap H^{\prime}\right) \leq 3$. Since $S \subset H^{\prime}$, we have $\operatorname{deg}\left(Z \cap H^{\prime}\right) \geq 2$. As in step (a) we get $\operatorname{deg}\left(Z \cap H^{\prime}\right)=3$ and that $Z \cap H^{\prime}$ is contained in an integral curve $T \subset Y$ with multidegree $\varepsilon_{i}$. Since $T$ has multidegree $\varepsilon_{i}, \pi_{i \mid T}$ is injective. Thus $S \nsubseteq T$, a contradiction.

Thus $\# \pi_{i}(S)=2$ for all $i$. Write $S=\{a, b\}$ and $Z=v \cup w$ with $v_{\text {red }}=\{a\}$ and $w_{\text {red }}=\{b\}$. Assume for the moment the existence of $i \in\{1, \ldots, k\}$ such that $\pi_{i \mid Z}$ is not an embedding. Since $\# \pi_{i}(S)=2$, there is one of the connected components of $Z$, say $v$, such that $\operatorname{deg}\left(\pi_{i}(v)\right)=1$ and there is $H \in\left|\mathcal{O}_{Y}\left(\varepsilon_{i}\right)\right|$ such that $H \cap Z=v$. Since $\mathcal{O}_{Y}(1, \ldots, 1)$ is very ample, $h^{1}\left(Y, \mathcal{I}_{v}(1, \ldots, 1)\right)=0$. Since $h^{1}\left(Y, \mathcal{I}_{Z}(1, \ldots, 1)\right)>0$, the residual exact sequence of $Y$ gives $h^{1}\left(Y, \mathcal{I}_{v}\left(\hat{\varepsilon}_{i}\right)\right)>0$. Since $\mathcal{O}_{Y_{i}}(1, \ldots, 1)$ is very ample, we get $\operatorname{deg}\left(\eta_{i}(w)\right)=1$, i.e. $\operatorname{deg}\left(\pi_{h}(w)\right)=1$ for all $h \neq i$. Exchanging the role of $w$ and $v$ we see that $(Y, Z)$ is as in case (a) of Example 4.2 and in particular $Y \cong \mathbb{P}^{1} \times \mathbb{P}^{1}$.

Assume that each $\pi_{i \mid Z}$ is an embedding. Thus there are $H \in\left|\mathcal{O}_{Y}\left(\varepsilon_{1}\right)\right|$ and $M \in\left|\mathcal{O}_{Y}\left(\varepsilon_{2}\right)\right|$ such that $H \cap Z=\{a\}$ and $M \cap Z=\{b\}$ (scheme-theoretic intersections). Thus $\operatorname{Res}_{H \cup M}(Z)=S$.

Claim 1: $k=2$.

Proof of Claim 1: Assume $k \geq 3$. Since $\operatorname{deg}(Z \cap(H \cup M))=2$ and $\mathcal{O}_{Y}(1, \ldots, 1)$ is very ample, $h^{1}\left(Y, \mathcal{I}_{Z \cap(H \cup M}(1, \ldots, 1)\right)=0$. Thus the residual exact sequence of $H \cup M$ gives $h^{1}\left(Y, \mathcal{I}_{S}(0,0,1, \ldots, 1)\right)$ $>0$. Since $\pi_{3 \mid S}$ is injective and $\# S=2, h^{1}\left(Y, \mathcal{I}_{S}\left(\varepsilon_{3}\right)\right)=0$. Thus $h^{1}\left(Y, \mathcal{I}_{S}(0,0,1, \ldots, 1)\right)=0$, a contradiction.

By Claim $1 Y=\mathbb{P}^{n_{1}} \times \mathbb{P}^{n_{2}}$.

Claim 2: $n_{1}=n_{2}=1$. 
Proof of Claim 2: Assume for instance $n_{1} \geq 2$. Thus there is $H \in\left|\mathcal{O}_{Y}\left(\varepsilon_{1}\right)\right|$ containing $S$. Since $S \subset H, H$ is a multiprojective space and $Y$ is the minimal multiprojective space containing $Z, 2 \leq$ $\operatorname{deg}(Z \cap H) \leq 3$.

First assume $\operatorname{deg}(Z \cap H)=2$, i.e. $S=Z \cap H$. Thus $S=\operatorname{Res}_{H}(Z)$. Since $\mathcal{O}_{H}(1,1)$ is very ample, $h^{1}\left(H, \mathcal{I}_{H \cap Z, H}(1,1)\right)=0$. The residual exact sequence (1) of $H$ gives $h^{1}\left(Y, \mathcal{I}_{S}(0,1)\right)>0$, contradicting the injectivity of $\pi_{2 \mid S}$.

Now assume $\operatorname{deg}(Z \cap H)=3$ and hence $\operatorname{deg}\left(\operatorname{Res}_{H}(Z)\right)=1$. Since $\mathcal{O}_{Y}(0,1)$ is globally generate and $\operatorname{deg}\left(\operatorname{Res}_{H}(Z)\right)=1, h^{1}\left(\mathcal{I}_{\operatorname{Res}_{H}(Z)}(0,1)\right)=0$. The residual exact sequence (1) of $H$ gives $h^{1}\left(H, \mathcal{I}_{Z \cap H, H}(1,1)\right)>0$. Since $\operatorname{deg}(Z \cap H)=3$ and $\nu_{1,1}(Z)$ is linearly dependent, $\nu_{1,1}(Z)$ spans a line, $L$. Since $X$ is scheme-theoretically cut-out by quadrics, $L \subset X$. Thus $L$ is a minimal degree subcurve of $X$. By Remark 4.1 there is $i \in\{1,2\}$ such that $\pi_{i}(L)$ is a point. Thus $\pi_{i}(Z \cap H)$ is a point. Since $S \subset Z \cap H$, \# $\pi_{i}(S)=1$, a contradiction.

By Claims 1 and 2 the pair $(Y, Z)$ is as in case (b) of Example 4.2 and in particular $Y \cong \mathbb{P}^{1} \times \mathbb{P}^{1}$. The uniqueness of the degree $\alpha$ containing $Z$ is obvious for $\alpha=1$, because two different lines intersects at at most one point. For $\alpha=2$ when $C$ is a smooth conic the uniqueness follows because in this case $\mathcal{O}_{Y}(1,1) \cdot \mathcal{O}_{Y}(1,1)=2$ (intersection number). The proofs in the last part of Example 4.2 show the uniqueness for reducible conics.

Proof of Theorem 1.3: Remark 4.1 shows that $\delta$ is the minimal degree of a subcurve of $X$ and describes all subcurves of degree $\delta$, proving (ii) and the second assertion of (i). Lemma 4.3 gives $\rho(X)^{\prime \prime}=\delta+1$. Part (iv) follows from the equality $\rho(X)^{\prime \prime}=\delta+1$ and Remark 4.1.

Since $\rho(X)^{\prime \prime}=\delta+1, \rho_{w}(X) \geq\lceil\delta / 2\rceil$. Since $X$ contains degree $\delta$ curves (Remark 4.1), $\rho_{w}(X) \leq$ $\lceil\delta / 2\rceil$ (Remark 2.9). Thus $\rho_{w}(X)=\lceil\delta / 2\rceil$ and taking $i \in E$, any curve $L \subset Y$ with multidegree $\varepsilon_{i}$ and any set $S \subset \nu_{d_{1}, \ldots, d_{k}}(L)$ with $\# S=\lceil\delta / 2\rceil$ we get an element of $\mathbb{L}(X)$. Taking unions $Z$ of arrows contained in $\nu_{d_{1}, \ldots, d_{k}}(L)$ and (if $\delta$ is odd) unions of arrows $Z$ with $\operatorname{deg}(Z)-\operatorname{deg}(Z \cap L)=1$, we also get elements of $\mathcal{L}(X)$ (Remark 4.1 of Proposition 3.2). If $\delta$ is even, then Lemma 4.4 proves part (v). Thus to prove the last unproved part of the theorem, i.e. part (vi), we may assume $\delta \geq 3$ and $\delta$ odd. We use the set-up of the proof of Lemma 4.4 with zero-dimensional schemes $A_{i}$, divisors $H_{i} \in\left|\mathcal{O}_{Y}\left(\varepsilon_{1}\right)\right|$, integers $e_{i}$ and the residual exact sequence (3). The only difference is that now $A_{0}:=A$ has degree $\delta+3$. Since the theorem is true in the case $k=1$, we may assume $\operatorname{dim} Y>1$ and that the theorem is true for all lower-dimensional projective space. Thus we may assume that $Y$ is the minimal multiprojective space containing $A$.

(a) In this step we assume the existence of some $i \in\left\{1, \ldots, d_{1}\right\}$ such that $h^{1}\left(H_{i}, \mathcal{I}_{A_{i-1} \cap H_{i}, H_{i}}\left(d_{1}-\right.\right.$ $\left.\left.i+1, d_{2}, \ldots, d_{k}\right)\right)>0$ and we call $g$ the first such an integer $i$. Since $\operatorname{dim} H_{i}<\operatorname{dim} Y$, the inductive assumption gives $e_{g} \geq 2+\min \left\{d_{1}-g+1, \delta\right\}$ and if equality holds $\nu_{d_{1}-g+1, d_{2}, \ldots, d_{k}}\left(A_{g-1}\right)$ is contained in a minimal degree curve of $\nu_{d_{1}-g+1, d_{2}, \ldots, d_{k}}\left(H_{i}\right)$. Since the sequence $\left\{e_{i}\right\}$ is non-increasing, we get $\delta+3 \geq 2 g+g \min \left\{d_{1}-g+1, \delta\right\}+\operatorname{deg}\left(A_{g}\right)$. Since $1 \leq g \leq d_{1}$ and $d_{1} \geq \delta \geq 3$, we get $g=1$, $d_{1}=\delta$ and $\operatorname{deg}\left(A_{g}\right) \leq 1$. Since $A \nsubseteq H_{1}$ by assumption, $\operatorname{deg}\left(A_{1}\right)=1$. Thus $\operatorname{deg}\left(H_{1} \cap A\right)=\delta+2$. Since $h^{1}\left(H_{1}, \mathcal{I}_{A \cap H_{1}, H_{1}}\left(d_{1}, \ldots, d_{k}\right)\right)>0$, Lemma 4.4 gives $d_{1}=\delta$ and the existence of a degree $\delta$ curve $T \subset \nu_{d_{1}, \ldots, d_{k}}\left(H_{1}\right) \subset X$ such that $\operatorname{deg}(Z \cap T)=\delta+2$. 
(b) Assume $h^{1}\left(H, \mathcal{I}_{A_{i} \cap H_{i}, H_{i}}\left(d_{1}-i+1, d_{2}, \ldots, d_{k}\right)\right)=0$ for all $i=1, \ldots, d_{1}$. Since $h^{1}\left(\mathcal{I}_{A}\left(d_{1}, \ldots, d_{k}\right)\right)$ $>0$, using all exact sequences (3) for $i=1, \ldots, d_{1}$ we get $h^{1}\left(\mathcal{I}_{A_{d_{1}}}\left(0, d_{2}, \ldots, d_{k}\right)\right)>0$. Since $\mathcal{O}_{Y}\left(0, d_{2}, \ldots, d_{k}\right)$ is globally generated, $\operatorname{deg}\left(A_{d_{1}}\right) \geq 2$. Hence $e_{1} \geq \cdots \geq e_{d_{1}}>0$. We have

$$
e_{1}+\cdots+e_{d_{1}}+\operatorname{deg}\left(A_{d_{1}}\right)=\delta+3 .
$$

(b1) Assume $\operatorname{deg}\left(A_{d_{1}}\right)=2$. Since $h^{1}\left(\mathcal{I}_{A_{d_{1}}}\left(0, d_{2}, \ldots, d_{k}\right)\right)>0, \pi_{1}\left(A_{d_{1}}\right)$ is a single point (schemetheoretically) (Lemma 4.5). The maximality of the integer $e_{d_{1}}$ gives $e_{d_{1}} \geq 2$. Since $d_{1} \geq \delta$ and the sequence $\left\{e_{i}\right\}$ is non-increasing, (4) gives $2 \delta+2 \geq \delta+3$, a contradiction.

(b2) Now assume $\operatorname{deg}\left(A_{d_{1}}\right) \geq 3$. By (4) $d_{1}=\delta, e_{i}=1$ for all $i$ and $\operatorname{deg}\left(A_{d_{1}}\right)=3$. Since $h^{1}\left(\mathcal{I}_{A_{d_{1}}}\left(0, d_{2}, \ldots, d_{k}\right)\right)>0$ and $\operatorname{deg}\left(A_{d_{1}}\right)=3$, Lemma 4.5 shows that either $\pi_{1 \mid A_{d_{1}}}$ is not an embedding or $\operatorname{deg}\left(A_{d_{1}}\right)=3$ and there is $i \in\{2, \ldots, k\}$ such that $\pi_{i}\left(A_{d_{1}}\right)$ is is a single point, as a scheme. In the former case we get $e_{\delta} \geq 2$ by the maximality property of all $e_{i}$, a contradiction. In the latter case we make the construction of Lemma 4.4 using the $i$-th factor of $Y$ instead of the first one and get $e_{1} \geq 3$, concluding again by (4) (note that we proved the impossibility of case (a) using any of the factors of $Y)$.

\section{References}

[1] B. Ådlandsvik, Joins and higher secant varieties, Math. Scan. 61 (1987), 213-222.

[2] E. Ballico, On strange projective curves, Rev. Roum. Math. Pures Appl. 37 (1992), 741-745.

[3] E. Ballico, On the irreducibility of the Severi variety of nodal curves in a smooth surface, Arch. Math. (Basel) 113 (2019), 483-487.

[4] E. Ballico, A. Bernardi and P. Santarsiero, Terracini locus for three points on a Segre variety, arXiv:2012.00574.

[5] E. Ballico and L. Chiantini, On the Terracini locus of projective varieties, Milan Journal of Mathematics, (2021), to appear: DOI: 10.1007/s00032-020-00324-5.

[6] V. Bayer and A. Hefez, Strange plane curves, Comm. Algebra 19 (1991), no. 11, 3041-3059.

[7] A. Bernardi, A. Gimigliano and M. Idà, Computing symmetric rank for symmetric tensors, J. Symbolic. Comput. 46 (2011), 34-55.

[8] J. Buczyński and J.M. Landsberg, On the third secant variety, J. Algebraic Combin, 40 (2014) 475-502.

[9] K. Chandler, Hilbert functions of dots in linear general position, Proc. Conference on Zero-Dimensional Schemes (Ravello, 1992), 65-79, de Gruyter, Berlin, 1994.

[10] K. A. Chandler, Geometry of dots and ropes, Trans. Amer. Math. Soc. 347 (1995), no. 3, 767-784.

[11] K. A. Chandler, A brief proof of a maximal rank theorem for generic double points in projective space, Trans. Amer. Math. Soc. 353 (2000), no. 5, 1907-1920.

[12] C. Ciliberto and R. Miranda, Interpolations on curvilinear schemes, J. Algebra 203 (1998), no. 2, 677-678.

[13] Th. Dedieu, Comment on: On the irreducibility of the Severi variety of nodal curves in a smooth surface, by E. Ballico, Arch. Math. (Basel) 114 (2020), no. 2, 171-174.

[14] R. Hartshorne, Algebraic Geometry, Springer-Verlag, Berlin-Heidelberg-New York, 1977.

[15] A. Hefez, Nonreflexive curves, Compositio Math. 69 (1989), no. 1, 3-35. 
[16] A. Hefez and S. L. Kleiman, Notes on the duality of projective varieties, Geometry today (Rome, 1984), 143-183, Progr. Math., 60, Birkhäuser Boston, Boston, MA, 1985.

[17] A. Iarrobino and V. Kanev, Power sums, Gorenstein algebras, and determinantal loci, Lecture Notes in Mathematics, vol. 1721, Springer-Verlag, Berlin, 1999, Appendix C by Iarrobino and Steven L. Kleiman.

[18] M. Kemeny, The universal Severi variety of rational curves on K3 surfaces, Bull. London. Math. Soc. 45 (2013), no. $1,159-174$.

[19] S. L. Kleiman, Tangency and duality, Proceedings of the 1984 Vancouver conference in algebraic geometry, 163-225, CMS Conf. Proc., 6, Amer. Math. Soc., Providence, RI, 1986.

[20] D. Laksov, Indecomposability of restricted tangent bundles, in: Young tableaux and Schur functors in algebra and geometry (Toruń, 1980), pp. 221-247, Astérisque 87-88, Soc. Math. France, Paris, 1981.

[21] J.M. Landsberg, Tensors: Geometry and Applications Graduate Studies in Mathematics, Amer. Math. Soc. Providence, 128 (2012).

[22] J.Landsberg and L. Manivel, Construction and classification of complex simple Lie algebras via projective geometry, Selecta Math. (N.S.) 8 (2002), no. 1, 137-159.

[23] A. Mazzon, On a geometric method for the identifiability of forms, Boll.UMI 13 (2020), 137-154.

[24] J. Rathmann, The uniform position principle for curves in characteristic $p$, Math. Ann. 276 (1987), no. 4, 565-579. 